\title{
Gender balance and impact of role models in secondary science education
}

\author{
Alessandro Siani ${ }^{\star, 1}$, Megan McArthur ${ }^{1}$, Bethany Christine Hicks ${ }^{1}$ and Claudiu Dacin ${ }^{2}$ \\ ${ }^{1}$ School of Biological Sciences, University of Portsmouth, Portsmouth, UK \\ ${ }^{2}$ The Cowplain School, Cowplain, UK
}

*Corresponding Author: alessandro.siani@port.ac.uk

Keywords: Role models; Gender bias; Secondary education; High school; STEM; Curricula

\begin{abstract}
Exposure to inspirational and relatable role models is crucial towards fostering engagement of learners with scientific disciplines. However, the representation of scientists in popular culture is still far from being adequately gender- and race-inclusive. This study evaluated the gender balance and impact of scientific role models using a twopronged experimental approach. The gender balance was investigated in search engines, online databases, and school curricula. A survey was used to investigate English secondary students' awareness of role models and intentions to pursue further scientific studies.
\end{abstract}

Our findings revealed a widespread female underrepresentation amongst scientific role models in all the analysed online sources and in high school curricula provided by the three main English exam boards. The survey $(n=$ 356) revealed that students were considerably more likely to identify famous male than female scientists. While awareness of female role models was significantly associated with students' gender, this was not the case for male role models. A statistically significant correlation was also observed between the number of identified role models and intentions to pursue scientific studies in Key Stage 4 girls.

This study reinforces the urgency of ensuring school curricula and online resources provide a more modern and inclusive representation of scientists.

\section{Introduction}

\section{The STEM gender gap in education and employment}

The existence of a marked gender bias in STEM (Science, Technology, Engineering and Mathematics) education and workplaces has been acknowledged for decades. As reported in Chambers' seminal study, 'Draw-AScientist" tests conducted in American elementary schools between 1966 and 1977 revealed that less than $1 \%$ of participating pupils depicted a woman when asked to draw a scientist (Chambers, 1983). Although this statistic has improved since, with on average $28 \%$ of students drawing a female scientist in the 1985-2016 period, the STEM field is still largely male-dominated, and gender bias and stereotyping are still prevalent in it (Miller et al., 2018). Worryingly, a progressive attrition has been observed throughout the STEM educational and professional pipeline, whereby the proportion of women decreases with career progression. A cogent example of the "leaky pipeline" phenomenon is the observation of female underrepresentation in the field of chemistry in the UK (Royal Society of Chemistry, 2018). Women represent $44 \%$ of chemistry students at the undergraduate level, and only $39 \%$ at $\mathrm{PhD}$ level. Female representation further decreases in the progression from education to employment and onto senior academic positions, whereby 
women constitute only $29 \%$ of non-professorial staff and $9 \%$ of professors. While the "leaky pipeline" is an effective metaphor to model female attrition in an academic context, it has been argued that some of its assumptions are too simplistic to account for the diverse range of professional trajectories in the STEM field, particularly with regards to the different progression routes within individual subfields (Cannady et al., 2014).

Regardless of the paradigm used to model the STEM female attrition and the resulting gender gap, it is clear that they are underpinned by different combinations of determinants depending of the stage of the educational and professional pipeline (UNESCO, 2017).

While the gender gap is less evident in primary than in secondary or higher education, it has been observed that already at the primary level girls are exposed to educational and societal biases that erode their self-confidence and motivation to further engage in Science and Mathematics (Blažev et al., 2017; Penner \& Paret, 2008). Secondary education is a critical time with regards to the consolidation of negative gender bias and students' selfefficacy (Makarova et al., 2019). Ultimately, this results in a marked reduction in the number of girls choosing to pursue STEM subjects after compulsory education (Boaler et al., 2011; Siani \& Dacin, 2018). The most recent data from the Higher Education Statistics Agency (HESA) indicate that women represent $41 \%$ $(42 \%$ for undergraduates, $39 \%$ for postgraduates) of total enrolments in science subjects (defined in the Common Aggregation Hierarchy as "CAH01 through to $\mathrm{CAH} 13$ with the exception of CAH12-01-13 - Human geography") in UK universities (Higher Education Statistics Agency, 2020). At the individual subject level, wide discrepancies in gender balance can be seen among different STEM disciplines, where female students' representation ranges from 20\% (Engineering and technology/CAH10 and Computing/ $\mathrm{CAH} 11)$, to $81 \%$ (Psychology/CAH04) and $82 \%$ (Veterinary sciences/CAH05).

Upon transition onto employment in the STEM sector, many women experience what has been described as a "chilly environment" in male-dominated workplaces, whereby they encounter an unsupportive and, in some instances, hostile environment brought about by sexist comments, harassment, condescension and lack of confidence from colleagues and managers. The combination of educational, socio-economic and internalised barriers to their retention and promotion ultimately leads to women's progressive underrepresentation along their professional trajectory (Avolio et al., 2020).

\section{Role models}

The observation that, regardless of the subject, exposure to inspirational and relatable role models plays a key part in determining a person's educational and professional choices is widely acknowledged by scholars and laypeople alike.

In accordance to Morgenroth's "Motivational Theory of Role Modeling", role models are figures which may differ widely in terms of background and notoriety, but share the ability to influence learners' goals and motivation via three key functions: to act as behavioural models, to represent the possible, and to be inspirational (Morgenroth et al., 2015).

Individuals exposed to a high performing role model demonstrate a higher goal acceptance, satisfaction and performance than those who were exposed to a lower performing role model (Earley \& Kanfer, 1985).

Examples of STEM role models include worldfamous scientists (e.g. Albert Einstein, Marie Curie) and communicators (e.g. David Attenborough, Neil deGrasse Tyson), as well as fictional characters (e.g. Dr. Leonora Orantes played by Marion Cotillard in the 2011 film "Contagion", Dr. Emmett Brown played by Christopher Lloyd in the "Back to the future" trilogy).

Indeed, not all STEM role models are necessarily famous or widely recognised; many exert their influence within a limited social context such as family, school or workplace.

Exposure to role models, regardless of their prestige, gender and ethnicity, has a profound positive impact on aspirations and performance for both men and women. However, it has been suggested that this effect 
may be particularly pronounced in the case of women exposed to role models of their own gender, whereas gender-matched role models did not have an increased impact on men (Lockwood, 2006). Moreover, the combination of gender-matching and subject-matching has been shown to further improve motivation and performance: women attain better on a maths test when it is administered by an experienced female mathematician rather than by a genderor subject-unmatched person (Marx \& Roman, 2002). Similarly, students from Black, White, and Asian backgrounds showed increased maths attainment when taught by an ethnicitymatched teacher, independently from their teaching quality (Egalite et al., 2015).

These effects can be explained by the observation that the impact of a role model is underpinned by two key factors: the perceived relevance of the role model to the individual, and the believed attainability of the role model's success (Lockwood \& Kunda, 1997). An effective role model is one that is both competent and relatable; a figure that personifies an individual's agency, potential to overcome gender and race barriers, and ability to achieve a successful career despite them.

The "Stereotype Inoculation Model" has been proposed as an interpretive lens for the impact of role models within academic and professional social groups (Dasgupta, 2011). According to this model, successful and inspirational figures act as "social vaccines" to immunise peers against negative stereotypes, increase their self-efficacy and foster their feeling of belonging within the social group.

Exposure to a diverse range of role models relatable to students of every ethnicity and gender is paramount in fostering their engagement and aspirations. Role-model interventions carried out in secondary schools significantly improved girls' enjoyment of maths and aspirations to pursue a career in the STEM field, and decreased their perception of negative stereotypes (González-Pérez et al., 2020). On the other hand, several studies demonstrate that the stereotype of the white, bespectacled, middle-aged male scientist is still prevalent in popular culture and perception (Fort \& Varney, 1989; Scherz \& Oren, 2006).
Unsurprisingly, the stereotypical representation of scientists makes them less likely to be relatable to students with diverse personal and social backgrounds. Previous findings indicate that only $12 \%$ of secondary students $(21 \%$ of girls, $4 \%$ of boys) named a woman when asked to name an influential scientist (Siani et al., 2020). Building upon those observations, the present study sought to investigate the factors that underpin this stereotype by analysing the gender balance of scientific role models available to secondary students in school curricula and online resources.

\section{Aims}

This study was designed to address two principal aims:

1) To investigate the gender balance of scientific role models available in online resources and secondary school curricula.

2) To evaluate secondary students' awareness of scientific role models, and whether it correlates with their intentions to pursue further studies in the STEM field.

As will be described in more details in the following section, two distinct experimental strategies were used to address these aims. Data on the gender balance of role models were gathered via online searches and analysis of secondary school curricula. Data on the recognition and impact of role models on secondary students were collected via a survey carried out in an English secondary school.

\section{Methods}

\section{Ethical approval}

This study was carried out in accordance with the University of Portsmouth research ethics guidelines. Ethical approval (approval code BIOL-ETHICS \#010-2020) was obtained by the investigators prior to the start of the study. All participants were informed of the voluntary and anonymous nature of the survey, and of their right to withdraw from it at any time and without any repercussions. No information allowing the identification of individual participants was collected in the survey, and all data was stored and handled in accordance to the General Data Protection Regulation. 


\section{Web searches}

The Internet Movie Database (IMDb) website was searched using the genre "Documentary" and the category "Science" to access a list of science documentaries. The results were sorted by descending popularity, and the first 40 results were analysed by recording the gender of the narrator and, if present, of any person appearing on the documentary poster. A further search was carried out amongst Movies and TV shows using the keyword "Scientist". The results were sorted by descending popularity, and the first 50 results were analysed by recording the gender of any person appearing on the poster.

To assess the accessibility of science role models in popular search engines, the keywords "Scientist" and "Famous Scientist" were input in Google, Bing, Yahoo, Yandex and Aol using their respective "image search" function. For each search engine, the first 50 images in order of appearance were analysed by recording the gender of the person or people shown in them.

The Times Educational Supplement (TES) website hosts a major repository of publicly available teaching resources, widely used by primary and secondary educators worldwide. The TES Resources website was searched using the keyword "Scientist", and the first 10 freely accessible sets of resources (e.g. posters, worksheets, slideshows) in order of appearance were analysed by recording the gender of any scientists that were mentioned in them.

All web searches were carried out between September and October 2020.

\section{GCSE curricula}

Most English secondary schools subscribe to three main exam boards, namely Assessment and Qualifications Alliance (AQA), Oxford, Cambridge and RSA Examinations (OCR), and Edexcel.

All three boards offer two options for Science at the General Certificate of Secondary Education (GCSE) level, namely "Triple Science" and "Combined Science". In the former option, students study the three science subjects (Physics, Chemistry, Biology) separately, and achieve a GCSE in each subject at the end of the course. In the latter, students study all three subjects, but upon completion they only achieve a GCSE in two of them. The number of male and female scientists named in the Triple Science and Combined Science GCSE specifications was recorded for each of the three exam boards based on the version of their curricula available on their respective websites as of October 2020.

\section{Survey design and distribution}

The questionnaire used in this study (Table 1) comprised a mix of Likert-type and open-ended questions designed to obtain, respectively, quantitative and qualitative data on students' awareness of science role models and their engagement with science subjects. In addition, a checkbox question was provided to quantify students' recognition of famous scientists' names, as well as a match-up question to evaluate their understanding of what discovery each scientist is renowned for. The selection and gender-matching of famous scientists who have lived in different eras and worked on different subjects is inherently problematic due to the absence of objective and fair inclusion/exclusion criteria. For example, a criterion based on their mentions in school books and curricula would not be appropriate due (as will be discussed throughout this paper) to the scarcity of female representation in such media. Similarly, other frequently used metrics such as the number of peer-reviewed publications or media appearances cannot be fairly used to compare modern scientists with those living decades or centuries ago. As a result, a list of influential scientists across different ages and scientific disciplines was compiled by the investigators in a way deemed comparable in terms of scientific impact and visibility in the eyes of the general public, with specific regards to school-age students.

The online survey was set up using Google Forms and distributed by secondary science teachers as a part of their science lessons in The Cowplain School (Hampshire, UK). The surveys were completed in January 2021 across Key Stage 3 (KS3, year 7 to year 9 ) and Key Stage 4 (KS4, year 10 to year 11). At the time of the Survey, the school was using the AQA "Combined Science: Trilogy" specifications. 


\begin{tabular}{|c|c|}
\hline & Survey Questions \\
\hline $1^{*}$ & What gender do you identify as? (MCQ) \\
\hline $2^{*}$ & What year group are you in? (MCQ) \\
\hline 3 & Name the first scientist that comes to mind (Open ended) \\
\hline 4 & $\begin{array}{l}\text { Can you name a scientist that you have seen on TV (perhaps in the news or a } \\
\text { documentary)? (Open ended) }\end{array}$ \\
\hline 5 & $\begin{array}{l}\text { Can you name a film or TV series either about, or involving a scientist character? If so, } \\
\text { please also list the name of the character if you can remember it. (Open ended) }\end{array}$ \\
\hline $6^{*}$ & $\begin{array}{l}\text { Here is a list of twenty influential scientists throughout history. Tick any that you have } \\
\text { heard of: (Check box) }\end{array}$ \\
\hline 7 & $\begin{array}{l}\text { Below is a selection of } 10 \text { of the most well-known scientists from the above list, and a } \\
\text { short description of their work. Match the scientist to what you think they discovered. } \\
\text { (Match up) }\end{array}$ \\
\hline 8 & $\begin{array}{l}\text { Rank your three favourite subjects at school, with } 1 \text { being your most favourite. (Likert } \\
\text { scale) }\end{array}$ \\
\hline 9 & $\begin{array}{l}\text { Rank the three sciences (Chemistry, Biology, and Physics) in order of how much you } \\
\text { like them, with } 1 \text { being your most favourite (Likert scale) }\end{array}$ \\
\hline 10 & $\begin{array}{l}\text { How much do you agree with the following statement: "Girls are better at Art and History } \\
\text { while boys are better at Science and Maths" (Likert scale) }\end{array}$ \\
\hline $11^{*}$ & $\begin{array}{l}\text { How likely are you to choose to study a science subject at college once you leave } \\
\text { school? (Likert scale) }\end{array}$ \\
\hline 12 & What science role models do you personally look up to, and why? (Open ended) \\
\hline
\end{tabular}

Table 1 Questionnaire used in the survey. The questions marked with an asterisk are the ones discussed in this study.

\section{Statistical analysis}

The study population was divided into four groups according to students' gender (male, female) and educational Key Stage (KS3, KS4). Survey responses were analysed using IBM SPSS statistics 26. Owing to the categorical nature of the questions used in the survey, non-parametric tests were used throughout the study. Kruskal-Wallis tests were used to compare medians between the four groups. Kendall $\tau_{b}$ coefficients were calculated to investigate the correlation between students' role model identification scores and their intention to pursue further scientific studies after compulsory education. A significance cutoff of $p=0.05$ was used in all tests.

\section{Results}

\section{Gender balance of scientific role models in web searches}

Out of the 40 most popular science documentaries on the IMDb website (Figure 1), 33 were narrated by a male narrator and 5 by a female one, while 2 did not have a narrator. For 18 of the documentaries the poster included the depiction of a person, 15 of them being men and 3 women. Among the 50 most popular movies and TV shows tagged with the keyword "Scientist" on IMDb, a total of 126 people were depicted in the poster, 88 men and 38 women. 


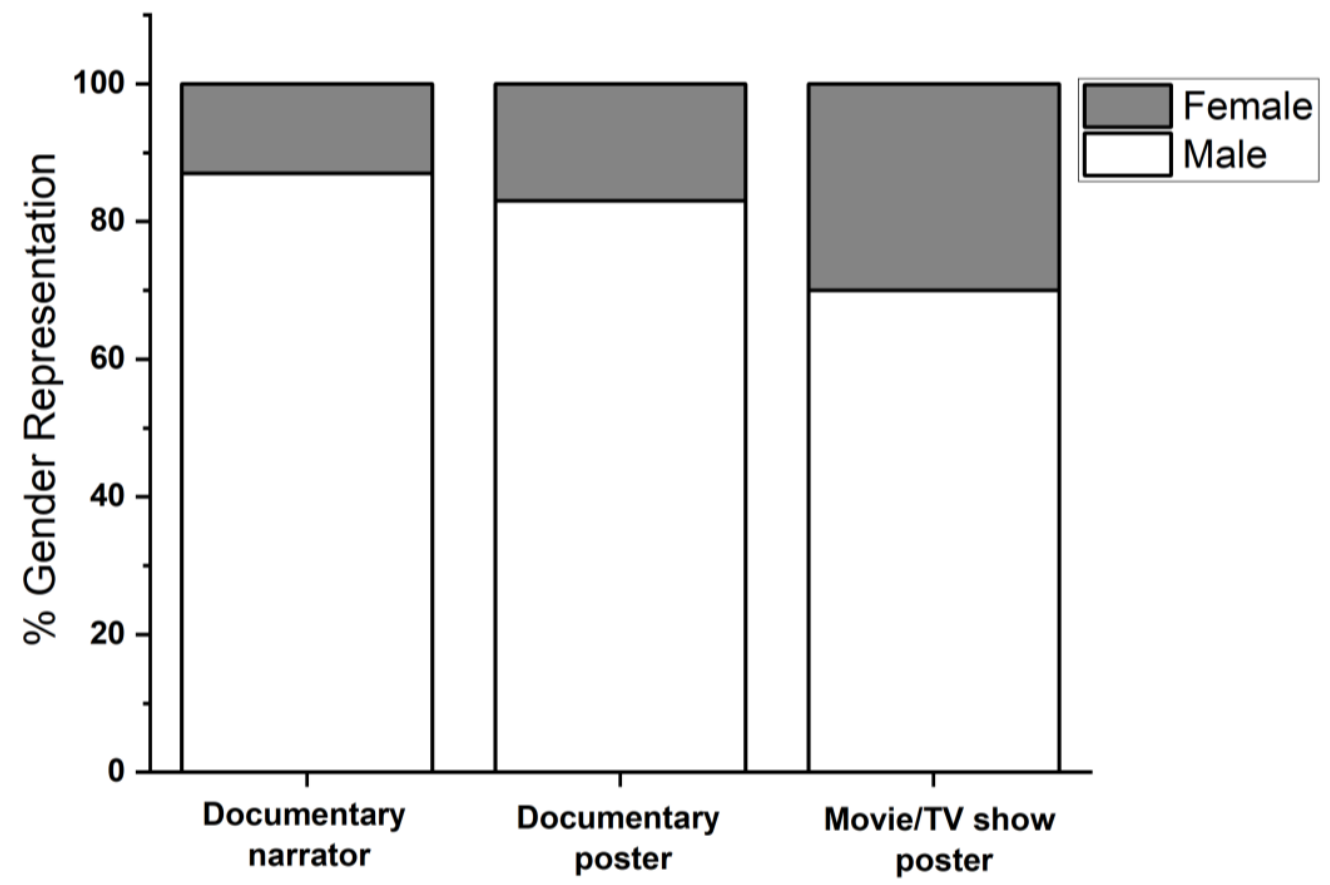

Figure 1 Gender balance of documentary narrators and people represented on the poster of documentaries, movies, and TV shows. Data retrieved from the IMDb website.

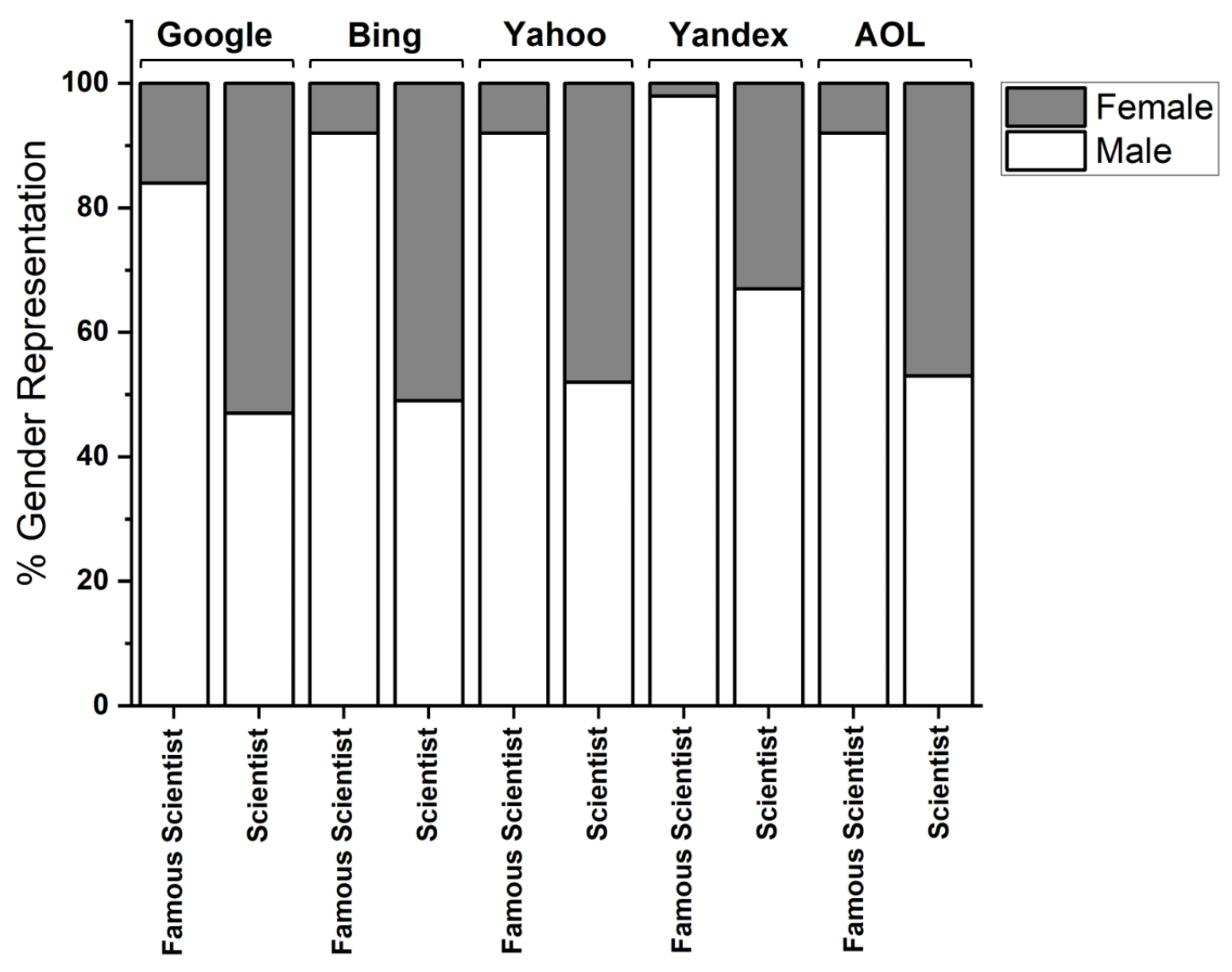

Figure 2 Gender balance in image searches carried out using 5 popular search engines. The keywords used in the searches were "Famous Scientist" and "Scientist". 


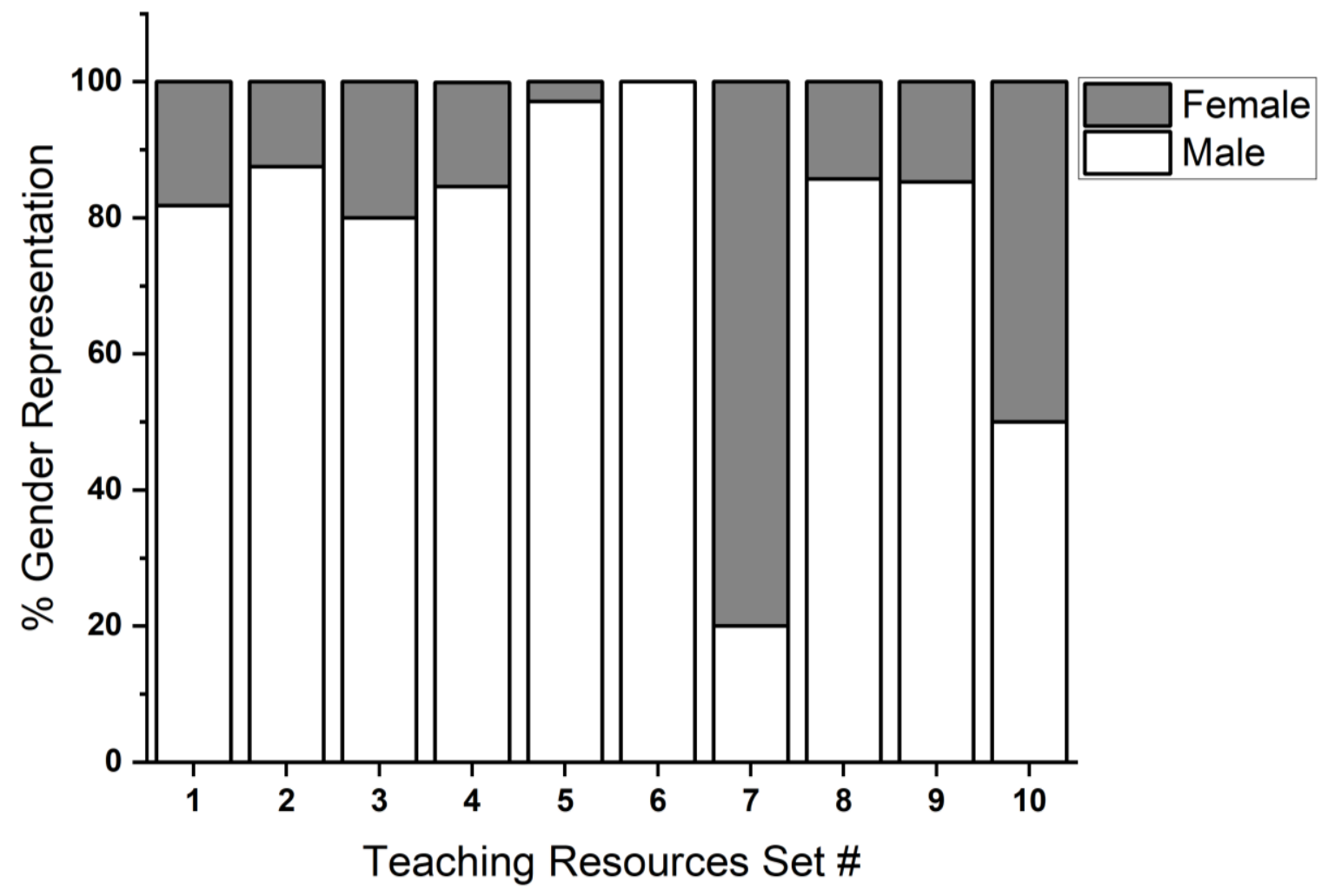

Figure 3 Gender balance of scientists mentioned in the first 10 freely available sets of teaching resources retrieved using the TES resources database.

Image searches carried out using different search engines using the keywords "Scientist" and "Famous scientist" revealed a widespread gender imbalance (Figure 2), particularly using the latter keywords. For both searches, the highest female representation was obtained via Google, and the lowest was obtained via Yandex.

In the first 50 images obtained using the keyword "Scientist", the female/male balance was 36/32 for Google, 28/27 for Bing, 27/29 for Yahoo, 25/28 for Aol, and 22/45 for Yandex. When using the keywords "Famous scientist", the female/male balance was 8/42 for Google, 4/46 for Aol, 4/46 for Bing, 4/46 for Yahoo, and $1 / 50$ for Yandex.

The first 10 sets of resources retrieved by searching for "Scientist" in the TES resources website were numbered in order of appearance and analysed individually for their gender balance (Figure 3). In increasing order of female representation, the female/male balance was $0 / 4$ in set \#6, 1/34 in set \#5, 2/14 in set \#2, 1/6 in set \#8, 21/122 in set \#9, 4/22 in set \#4, 2/9 in set \#1, 2/8 in set \#3, 15/15 in set \#10, and $8 / 2$ in set \#7. In total, 56 female and 236 male scientists were named in the 10 sets of resources retrieved on TES, corresponding to an overall gender balance of over $4: 1$ in favour of men.

\section{Gender balance of scientists mentioned in GCSE curricula}

The AQA, Edexcel and OCR GCSE Science specifications were analysed to assess the gender balance of any scientists mentioned by name in the curricula. The gender balance was analysed separately for each of the three sciences (Biology, Physics, Chemistry) for the Triple Science specifications, and cumulatively for the Combined Science specifications (Figure 4). No female scientists were mentioned in any of the Physics curricula, while 7 males were named in the OCR specifications, 4 for AQA, and 2 for Edexcel. In Biology, no women and 7 men were mentioned in the AQA specifications, and only one woman was named in the Edexcel and OCR specifications, as opposed to, respectively, 3 and 6 men. 


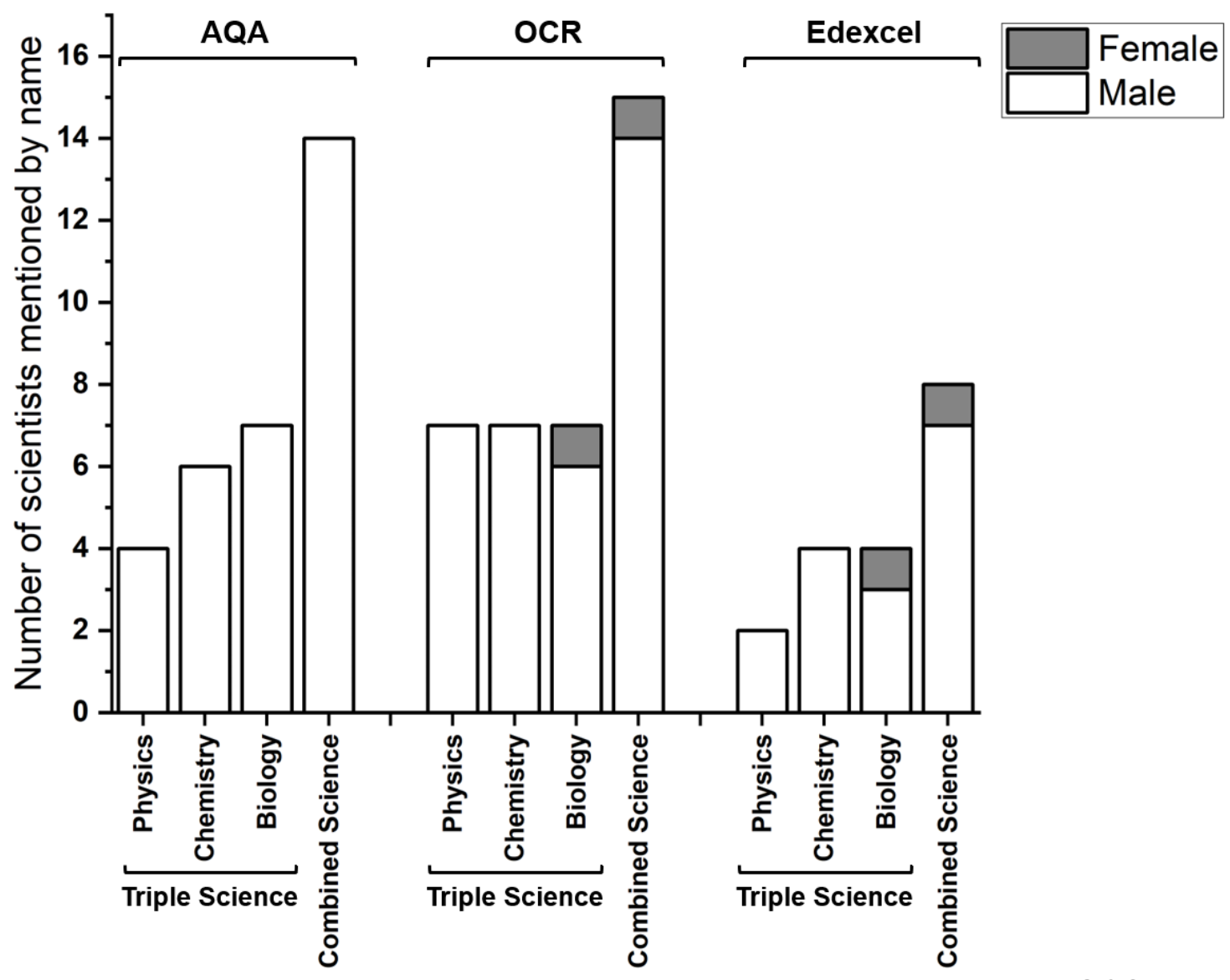

Figure 4 Number of female and male scientists mentioned by name in the GCSE specifications provided by the AQA, OCR, and Edexcel exam boards.

\begin{tabular}{|l|c|c|c|c|}
\hline & KS3 & KS4 & No answer & Total by gender \\
\hline Female & 99 & 81 & 4 & 184 \\
\hline Male & 92 & 66 & 1 & 159 \\
\hline Other/No answer & 6 & 5 & 2 & 13 \\
\hline Total by key stage & 197 & 152 & 7 & \\
\hline
\end{tabular}

Table 2 Breakdown of the survey population by gender (rows) and educational key stage (columns).

No women were mentioned in any of the Chemistry specifications, whereas 7 men were named in the OCR curriculum, 6 in AQA, and 4 in Edexcel.

In the Combined Science specifications, no women and 14 men were named for AQA, 1 woman and 11 men for OCR, and 1 woman and 7 men for Edexcel.

\section{Survey participants}

The study included a total of 356 high school students, roughly evenly distributed by gender (44.7\% male, $51.7 \%$ female) and educational key stage $(55.3 \% \mathrm{KS} 3,42.7 \% \mathrm{KS} 4)$. The full breakdown of the study population by gender and key stage is provided in Table 2 .

\section{Awareness of scientific role models among secondary school students}

When asked to identify famous scientists from a list, students recognised male scientists much more frequently than female scientists. Out of the 20 names provided, men represented the 4 most frequently identified scientists, and women the 6 least frequently identified ones (Figure 5). None of the 10 female scientists was recognised by more than $50 \%$ students, and 8 of them were only recognised by less than $20 \%$ of the students. 


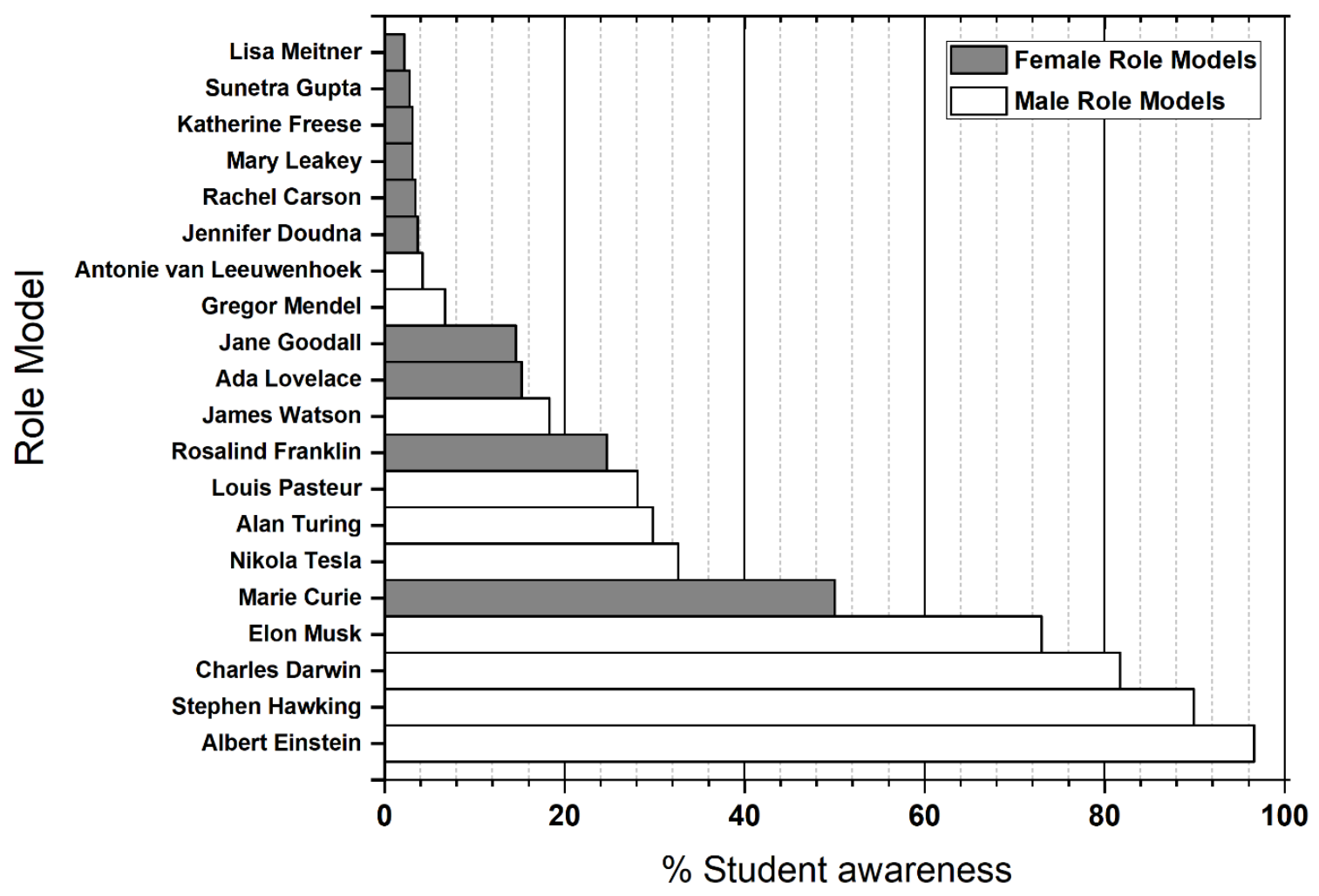

Figure 5 Awareness of female and male scientific role models amongst the high school students who participated in the survey.

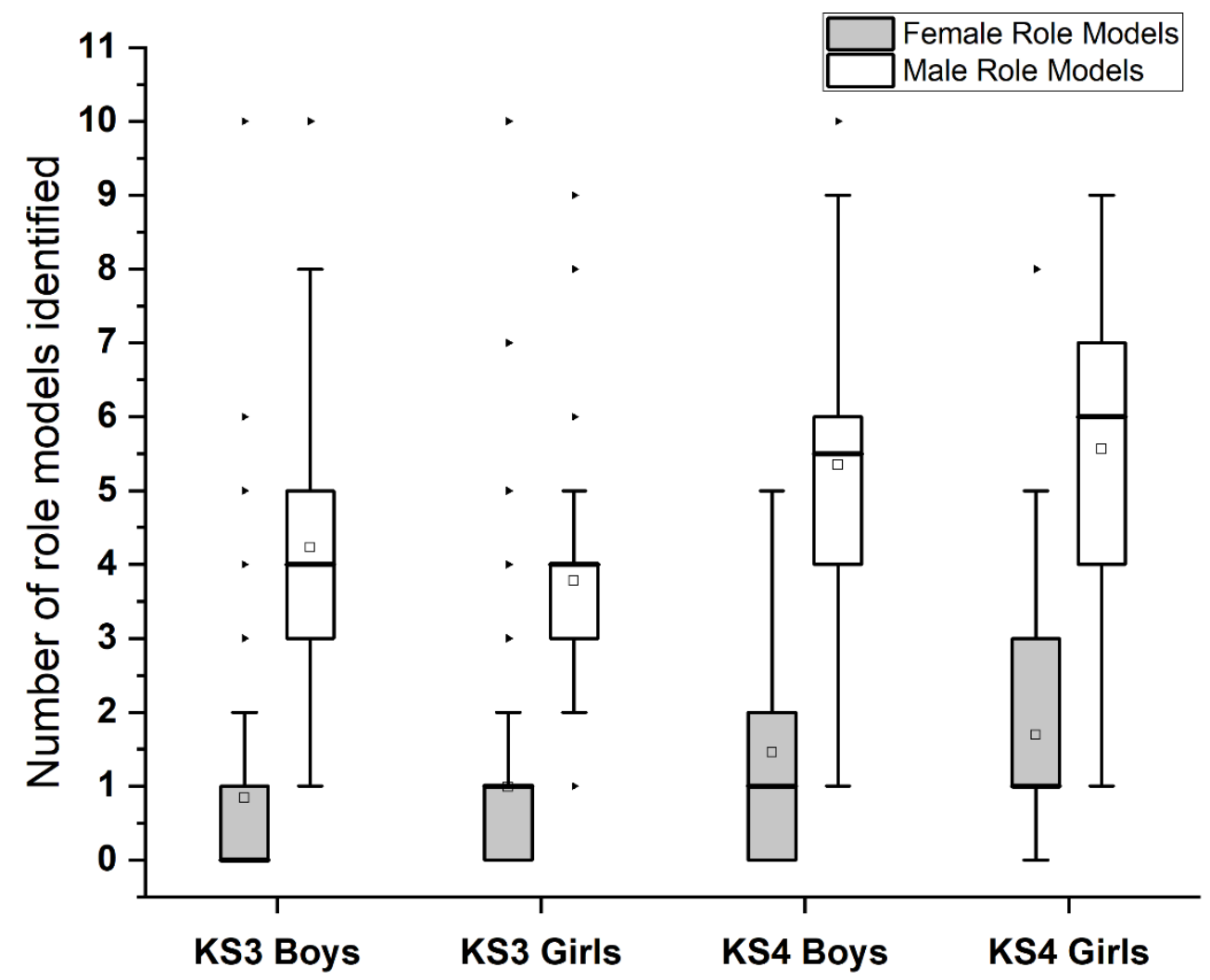

Figure 6 Number of female and male scientific role models identified by high school students grouped by their gender and key stage. 


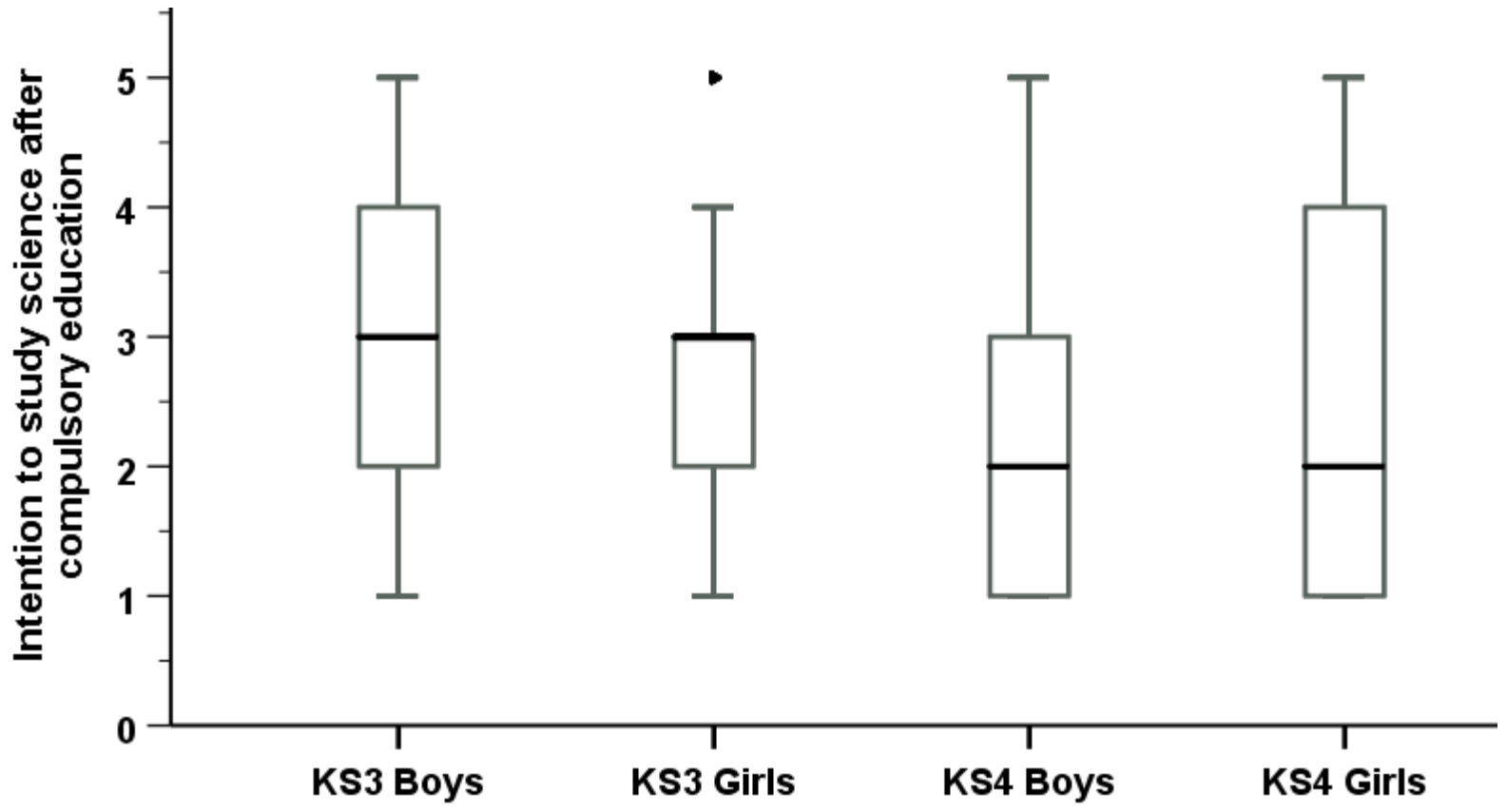

Figure 7 Students' self-reported likelihood to study science after compulsory education on a scale from 1 (highly unlikely) to 5 (highly likely).

Male role models (median range 4-6) were identified considerably more frequently than female role models (median range 0-1) regardless of the gender and key stage of the survey participants (Figure 6).

The median number of identified male role models was significantly different by students' key stage $\left(\chi^{2}=59.835 ; d f=1 ; p=1.03 \times\right.$ $\left.10^{-14}\right)$, but not by their gender $\left(\chi^{2}=0.494\right.$; $d f=1 ; p=0.482$ ).

However, the median number of identified female role models was significantly different both by students' key stage $\left(\chi^{2}=30.424 ; d f=\right.$ $\left.1 ; p=3.47 \times 10^{-8}\right)$, and their gender $\left(\chi^{2}=\right.$ $4.099 ; d f=1 ; p=0.043)$.

\section{Intention to study science after compulsory education}

Students' intentions to study a scientific subject after compulsory education are shown in Figure 7. Key Stage 3 students were significantly more likely $\left(\chi^{2}=17.637 ; d f=1\right.$; $p=0.000027$ ) to express an intention to pursue further scientific studies than Key Stage 4 students. There was no significant association $\left(\chi^{2}=0.715 ; d f=1 ; p=0.398\right)$ between students' gender and their intention to pursue further scientific studies.

For Key Stage 3 boys, Kendall $\tau_{b}$ coefficient showed no correlation between number of gender-matched $\left(\tau_{b}=0.077 ; p=0.360\right)$ or gender-unmatched $\left(\tau_{b}=0.158 ; \quad p=0.078\right)$ role models identified and intentions to pursue further scientific studies. For Key Stage 3 girls, no correlation was observed between number of gender-matched $\left(\tau_{b}=0.115 ; p=0.181\right)$ or gender-unmatched $\left(\tau_{b}=0.153 ; p=0.065\right)$ role models identified and intentions to pursue further scientific studies. For Key Stage 4 boys, no correlation was observed between number of gender-matched $\left(\tau_{b}=-0.004 ; p=0.971\right)$ or gender-unmatched $\left(\tau_{b}=0.134 ; p=0.197\right)$ role models identified and intentions to pursue further scientific studies. For Key Stage 4 girls, a statistically significant correlation was observed between number of gender-matched $\left(\tau_{b}=0.278 ; p=0.002\right)$ or gender-unmatched $\left(\tau_{b}=0.242 ; p=0.007\right)$ role models identified and intentions to pursue further scientific studies. 


\section{Discussion}

The underrepresentation of women in STEM careers and their progressive attrition along the educational and professional pipeline have profound social and economic repercussions.

The waste of human potential caused by gender bias and "glass ceilings" contributes to the STEM skills shortage at a time when economic and human investment in research and innovation is crucial to rise up to increasingly pressing global challenges such as (to name a few) climate change, cybersecurity, population aging, and transmissible diseases.

The European Institute for Gender Equality estimated that closing the gender gap in STEM education and employment could lead to the creation of up to 1.2 million new jobs across the EU and to a 2.2-3\% improvement in GDP per capita, corresponding to a GDP improvement of $€ 610-820$ billion by 2050 (European Institute for Gender Equality, 2017).

Addressing the gender gap requires not only the removal of any socio-economic barriers precluding access, retention and progression along the STEM pipeline, but also a systematic mentality overhaul to overcome longengrained beliefs and stereotypes.

The analysis of gender balance in sciencethemed educational and recreational resources provides a striking example of the prevalence of stereotypes in the public perception of the scientist figure. Our findings indicate that, while image searches for "Scientist" provide relatively gender-balanced results (female representation 33-53\%) across most search engines, image searches for "Famous scientist" only returned a small minority $(2-16 \%)$ of women. Such a marked difference in search results obtained by just adding the adjective "famous" to the search string highlights an interesting aspect of the public perception of scientists. Searching for "Scientist" mostly yields generic stock photos that take into account the gender and race diversity of the profession, often depicting models rather than "real-life" scientists.

On the other hand, the key phrase "Famous scientist" mostly returns portraits of notable historical figures (typically Albert Einstein, Charles Darwin, Isaac Newton, Leonardo da Vinci, etc.) who lived at times when scientific research was by and large a prerogative of white males. However, a marked gender imbalance can be observed even in the relatively rare occurrences when modern scientists are represented in search results, whereby famous male scientists (e.g. Stephen Hawking, Brian Cox, etc.) are more frequently depicted than their female counterparts (e.g. Nobel Prizes Emmanuelle Charpentier and Jennifer Doudna).

A similar trend was unearthed by the analysis of gender balance in documentaries, films, and TV shows accessible on the IMDb website, where women represented less than $30 \%$ of the figures depicted on the promotional posters. Worryingly, not only are female scientists considerably underrepresented in popular media, but their depiction often reinforces sexist stereotypes by focusing on their attractiveness and romantic relationships rather than their scientific accomplishments (Steinke \& Paniagua Tavarez, 2017).

In addition to the gender-imbalanced representation of scientists in search engines and visual entertainment media, our findings revealed a marked underrepresentation of women in the educational context. Of the first 10 sets of teaching resources retrieved from the TES website using the keyword "Scientist", only one represented an equal number of male and female scientists; another set showed more women than men, however female representation in the remaining 8 sets was less than $25 \%$. Worryingly, an overwhelming gender imbalance was observed in the GCSE Science specifications provided by the three main English exam boards. In total, 31 men and no women were mentioned across the Triple Science and Combined Science AQA curricula. In the OCR curricula, there were 34 mentions of male scientists and only 2 of a female scientist (Rosalind Franklin was mentioned in both the Biology and Combined Science specifications). In the Edexcel curricula, there were 16 mentions of male scientists and only two of a female scientist (Mary Leakey was mentioned in both the Biology and Combined Science specifications). 
The content of high school science curricula has been identified as a critical determinant of students' experience, motivation, and desire to pursue a career in the field (Shirazi, 2017). In that respect, the observation of the striking gender imbalance in UK high school specifications reinforces the urgent need to update school curricula and provide students with a balanced and inclusive representation of science and scientists.

The results of our survey indicate that, regardless of their gender and Key Stage, students are overwhelmingly more likely to recognise male scientists than female ones. Out of 20 names provided, men represented the four most frequently recognised scientists, and women the six least frequently recognised ones. All female role models were only recognised by less than $25 \%$ of students, with the exception of Marie Curie who was recognised by $50 \%$ of them. Key Stage 4 students were significantly more able than Key Stage 3 students to identify scientists of both genders, reflecting their higher educational level. No statistically significant association was observed between students' gender and their ability to recognise male or female role models. Interestingly, students' responses highlighted that their intention to study a scientific subject after compulsory education decreases significantly from Key Stage 3 to Key Stage 4. A tentative interpretation for this observation is that younger students may have a more romanticised view of science and be less aware of the challenges inherent to the subject. This explanation is supported by a recent longitudinal study suggesting that students' intentions to pursue scientific studies are strongly affected by the perceived easiness of the subject (Potvin et al., 2020).

Our survey unearthed a statistically significant association between the number of role models identified by Key Stage 4 girls and their intention to pursue further scientific studies.

This finding corroborates previous observations that exposure to charismatic and relatable role models has a positive impact on students' self-efficacy, motivation and ambition to pursue a career in their subject (Morgenroth et al., 2015). However, there is no clear literature consensus on whether gendermatched role models have a stronger impact on girls' aspirations and performance (Lockwood, 2006; Porter \& Serra, 2020) or this inspirational effect manifests itself regardless of gender-matching (Carrington et al., 2008; Conner \& Danielson, 2016). By showing that awareness of scientific role models, regardless of their gender, has a significant correlation with girls' intentions to pursue further scientific studies, the results of our survey indicate that the latter hypothesis holds true for the population surveyed in the present study.

\section{Conclusions}

Students' perception of scientists is principally influenced by two key factors, namely their depiction in popular media and in science school lessons (Tan et al., 2017).

The present study highlighted that, despite decades of research and interventions aimed at mitigating gender bias in the STEM field (Hyde et al., 2009; Wang \& Degol, 2017), scientific role models available in recreational and educational contexts are still far from being gender-balanced. The analysis of online resources (search engines, IMDb, TES) and Science GCSE specifications (AQA, Edexcel, OCR) revealed that scientists are represented in an overwhelmingly gender-exclusive manner. The survey carried out in an English secondary school as a part of this study confirmed that students' awareness of scientific role models is still largely maledominated. However, given the lack of objective inclusion/exclusion and gendermatching criteria in the selection of the influential scientists used in the survey, it cannot be ruled out that the survey outcomes might vary if different sets of scientists were chosen.

While the present study gives a comprehensive account of the gender-balance of scientific role models in online sources and high school curricula, further investigations would be required to investigate gender balance in other influential sources (e.g. books, video games, YouTube channels). Another limitation of the study is that the survey was carried out in a single secondary school, meaning that the outcomes may only partially apply to students belonging to different socio-economic and cultural contexts. 
In conclusion, this study reinforces the need for policy makers and educational regulatory bodies to take urgent action to ensure that school curricula and didactic materials represent scientists in a gender-balanced and inclusive fashion, reflecting the diversity and inclusivity endeavoured in modern academic and professional settings.

\section{Funding}

The authors received no specific funding for this work.

\section{Conflict of interest}

The authors declare that they have no conflict of interest.

\section{Availability of data and materials}

The dataset analysed in this study is available from the corresponding author on reasonable request.

\section{References}

Avolio, B., Chavez, J., \& Vilchez-Roman, C. (2020). Factors that contribute to the underrepresentation of women in science careers worldwide: a literature review. Social Psychology of Education. DOI: 10.1007/s11218-020-09558-y

Blažev, M., Karabegović, M., Burušić, J., \& Selimbegović, L. (2017). Predicting genderSTEM stereotyped beliefs among boys and girls from prior school achievement and interest in STEM school subjects. Social Psychology of Education, 20(4), 831-847. DOI: 10.1007/s11218-017-9397-7

Boaler, J., Altendorff, L., \& Kent, G. (2011). Mathematics and science inequalities in the United Kingdom: when elitism, sexism and culture collide. Oxfood Review of Education. (4), $457 . \quad$ DOI: $10.1080 / 03054985.2011 .595551$

Cannady, M.A., Greenwald, E., \& Harris, K.N. (2014). Problematizing the STEM Pipeline Metaphor: Is the STEM Pipeline Metaphor Serving Our Students and the STEM
Workforce? Science Education, 98(3), 443460. DOI: $10.1002 /$ sce. 21108

Carrington, B., Tymms, P., \& Merrell, C. (2008). Role Models, School Improvement and the 'Gender Gap'-Do Men Bring out the Best in Boys and Women the Best in Girls? British Educational Research Journal, 34(3), 315-327. DOI: $10.1080 / 01411920701532202$

Chambers, D.W. (1983). Stereotypic Images of the Scientist: The Draw-A-Scientist Test. Science Education, 67(2), 255-265. DOI: $10.1002 /$ sce. 3730670213

Conner, L.D.C. \& Danielson, J. (2016). Scientist Role Models in the Classroom: How Important Is Gender Matching? International Journal of Science Education, 38(15), 24142430. DOI: 10.1080/09500693.2016.1246780

Dasgupta, N. (2011). Ingroup Experts and Peers as Social Vaccines Who Inoculate the Self-Concept: The Stereotype Inoculation Model. Psychological Inquiry, 22(4), 231-246. DOI: 10.1080/1047840X.2011.607313

Earley, P.C., \& Kanfer, R. (1985). The influence of component participation and role models on goal acceptance, goal satisfaction, and performance. Organizational Behavior and Human Decision Processes, 36(3), 378-390. DOI: 10.1016/0749-5978(85)90006-8

Egalite, A.J., Kisida, B. \& Winters, M.A. (2015). Representation in the classroom: The effect of own-race teachers on student achievement. Economics of Education Review, 45, 44-52. DOI: 10.1016/j.econedurev.2015.01.007

European Institute for Gender Equality. (2017). Economic benefits of gender equality in the EU How gender equality in STEM education leads to economic growth.

Fort, D.C. \& Varney, H.L. (1989). How Students See Scientists: Mostly Male, Mostly White, And Mostly Benevolent. Science and Children, 26(8), 8-13.

González-Pérez, S., Mateos de Cabo, R. \& Sáinz, M. (2020). Girls in STEM: Is It a Female Role-Model Thing? Frontiers in Psychology, 11. DOI: $10.3389 / f p s y g .2020 .02204$ 
Higher Education Statistics Agency. (2020). What do HE students study? Retrieved 18/06/2021 from https://www.hesa.ac.uk/dataand-analysis/students/what-study

Hyde, J.S., Mertz, J.E. \& Schekman, R. (2009). Gender, Culture, and Mathematics Performance. Proceedings of the National Academy of Sciences of the United States of America, 106(22), 8801-8807. DOI: 10.1073/pnas.0901265106

Lockwood, P. (2006). "Someone Like Me Can Be Successful": Do College Students Need Same-gender Role Models? Psychology of Women Quarterly, 30(1), 36-46. DOI: 10.1111/j.1471-6402.2006.00260.x

Lockwood, P. \& Kunda, Z. (1997). Superstars and Me: Predicting the Impact of Role Models on the Self. Journal of Personality and Social Psychology, 73(1), 91-103. DOI: 10.1037/0022-3514.73.1.91

Makarova, E., Aeschlimann, B. \& Herzog, W. (2019). The Gender Gap in STEM Fields: The Impact of the Gender Stereotype of Math and Science on Secondary Students' Career Aspirations. Frontiers in Education, 4. DOI: 10.3389/feduc.2019.00060

Marx, D.M. \& Roman, J. S. (2002). Female role models: Protecting women's math test performance. Personality and Social Psychology Bulletin, 28(9), 1183-1193. DOI: $10.1177 \% 2 F 01461672022812004$

Miller, D.I., Nolla, K.M., Eagly, A.H. \& Uttal, D.H. (2018). The Development of Children's Gender-Science Stereotypes: A Meta-analysis of 5 Decades of U.S. Draw-A-Scientist Studies. Child Development, 89(6), 1943-1955. DOI: 10.1111/cdev.13039

Morgenroth, T., Ryan, M.K. \& Peters, K. (2015). The motivational theory of role modeling: How role models influence role aspirants' goals. Review of General Psychology, 19(4), 465-483. DOI: 10.1037/gpr0000059

Penner, A.M. \& Paret, M. (2008). Gender differences in mathematics achievement: Exploring the early grades and the extremes.
Social Science Research, 37(1), 239-253. DOI: 10.1016/j.ssresearch.2007.06.012

Porter, C. \& Serra, D. (2020). Gender differences in the choice of major: The importance of female role models. American Economic Journal: Applied Economics, 12(3), 226-254. DOI: 10.1257/app.20180426

Potvin, P., Hasni, A., Sy, O. \& Riopel, M. (2020). Two Crucial Years of Science and Technology Schooling: A Longitudinal Study of the Major Influences on and Interactions between Self-Concept, Interest, and the Intention to Pursue S\&T. Research in Science Education, 50(5), 1739-1761. DOI: $10.1007 / \mathrm{s} 11165-018-9751-6$

Royal Society of Chemistry. (2018). Breaking the Barriers Women's retention and progression in the chemical science.

Scherz, Z. \& Oren, M. (2006). How to Change Students' Images of Science and Technology. Science Education, 90(6), 965-985. DOI: 10.1002/sce.20159

Shirazi, S. (2017). Student Experience of School Science. International Journal of Science Education, 39(14), 1891-1912. DOI: 10.1080/09500693.2017.1356943

Siani, A. \& Dacin, C. (2018). An Evaluation of Gender Bias and Pupils' Attitude towards STEM Disciplines in the Transition between Compulsory and Voluntary Schooling. New Directions in the Teaching of Physical Sciences, 13(1), n1. DOI: 10.29311/ndtps.v0i13.2966

Siani, A., Marley, S.A., Smith, C. \& Donnelly, J. (2020). Gender and parental education as indicators of students' engagement with STEM subjects. International Journal of Gender, Science and Technology, 12(2).

Steinke, J. \& Paniagua Tavarez, P. M. (2017). Cultural Representations of Gender and STEM: Portrayals of Female STEM Characters in Popular Films 2002-2014. International Journal of Gender, Science \& Technology, 9(3), 244.

Tan, A.L., Jocz, J.A. \& Zhai, J. (2017). Spiderman and science: How students' 
Gender balance and impact of role models in secondary science education

perceptions of scientists are shaped by popular media. Public Understanding of Science, 26(5), 520-530. DOI: $10.1177 / 0963662515615086$

Wang, M.T. \& Degol, J.L. (2017). Gender Gap in Science, Technology, Engineering, and Mathematics (STEM): Current Knowledge, UNESCO. (2017). Cracking the code: Girls' and women's education in STEM. Implications for Practice, Policy, and Future Directions. Educational Psychology Review (1), 119. DOI: 10.1007/s10648-015-9355-x 